\title{
Neurocritical Care in Canada: Evolving Streams in a New Discipline
}

\author{
Eyad Al Thenayan, Charles Bolton, Draga Jichici, Martin Savard, Jeanne Teitelbaum, \\ Bryan Young, David Zygun
}

Can. J. Neurol. Sci. 2008; 35: 405-408

Neurocritical care is a branch of intensive care medicine devoted to life-threatening diseases of the nervous system. ${ }^{1}$ The Table lists various disorders of the central and peripheral nervous systems that are encountered, either as primary neurological/neurosurgical disorders or as complications of systemic diseases or their therapy.

Neurocritical care developed as a national discipline in Canada with the founding of the Canadian Neurocritical Care Group in 1992. Members were almost exclusively from the neurology and neurosurgery communities. The Group developed an annual course held concurrently with the Canadian Congress of Neurological Sciences and revised the guidelines for the determination of brain death in $1999 .^{2}$ These have subsequently been revised by the Canadian Council for Donation and Transplantation in 2003. This development reflected the increasing interest of intensivists with special training in neurocritical care to be involved and lead to the founding of the Canadian Neurocritical Care Society in 2003. By-laws were drafted and annual meetings have been held in conjunction with the Critical Care Canada Forum. The founding and still present co-chairs are Drs. Draga Jichici and David Zygun. At present there are 49 members. The majority (61\%) have fellowship training in critical care medicine. Of those with critical care medicine fellowship training, internal medicine $(51 \%)$ is the most common base specialty followed by anaesthesia (19\%), pediatrics $(11 \%)$, neurology $(7 \%)$, surgery $(7 \%)$ and emergency medicine (4\%). Of those who have not completed formal critical care medicine training, 11 are neurologists or neurologists in training, 4 are neurosurgeons and 2 are anesthetists. The Society exists to set practice guidelines and standards of care, ${ }^{3}$ develop standards of training and education for neurocritical care medicine, promote and perform research with the goal of improving outcome from life-threatening neurological illness and advocate for the profession.

\section{Rationale for Neurocritical Care}

We wish to put forward two main theses:

1. An organized approach with special training, experience and protocols for treating various conditions produces improved outcomes and is cost-effective.

Diringer and Edwards conducted an analysis of data from 42 intensive care units (ICUs), including one NICU staffed by neurologists, and evaluated factors related to mortality in victims of spontaneous intracerebral hemorrhage (ICH). ${ }^{4}$ They found that admission to an NICU was associated with a significant reduction in in-hospital mortality, compared with those admitted to general ICUs, even after adjusting for severity of illness and other variables. It is important to note a large proportion of the general ICUs forming the comparison group did not have full time fellowship trained intensivist coverage, weakening the generalizability of these results to the Canadian model. Although there are no specific, single aspects of treatment for ICH (e.g. surgical removal of clot or intracranial pressure (ICP) monitoring) that have proven to be effective, the overall expertise of management of neurological issues by specially trained doctors and nurses appears to offer an advantage in patient outcomes.

The introduction of a neurointensivist into an existing NICU, with leadership, a team approach, nursing training, protocols, better documentation and improved monitoring systems, was followed by decreased NICU mortality, shorter hospital length of stay and improved outcomes. ${ }^{5}$ Thus, as with stroke units, the programmatic team approach is probably more important than just having an NICU.

Elf and colleagues conducted a before-and-after observational study in their NICU in Sweden. ${ }^{6}$ There was a significant reduction in mortality after the institution of careful monitoring with protocols directed towards managing and preventing secondary brain damage in head-injured patients. A similar retrospective before-and-after project by Patel and colleagues showed that the introduction of neurointensive care performed collaboratively by dedicated neurointensivists, neuroanethetists and neurosurgeons was associated with significantly better outcomes in patients with severe traumatic brain injury. ${ }^{7}$ Another showed that patients with acute traumatic

From the Critical Care Program (EAT), Departments of Clinical Neurological Sciences and Medicine (BY), University of Western Ontario, London, Ontario; Department of Medicine (CB), Queen's University, Kingston, Ontario; Divisions of Neurology and Critical Care Medicine (DJ), McMaster University, Hamilton, Ontario; Department of Medicine (MS), Laval University, Quebec; Department of Medicine (JT), McGill University, Montreal, PQ; Department of Critical Care Medicine (DZ), University of Calgary, Calgary, Alberta, Canada.

Received September 18, 2007. Final Revisions Submitted March 31, 2008. Correspondence to: Draga Jichici, 85 Norfolk St., Guelph, Ontario, N1H 4J4, Canada. 


\section{Table: Conditions Appropriate for Neurocritical Care}

\section{Primary Nervous System Disorders}

Epilepsy

Stroke

Status epilepticus and related conditions

Ischemic stroke, especially with impaired consciousness Hemorrhagic Stroke: Intraparenchymal hemorrhages

Subarachnoid hemorrhage with complications and impaired consciousness, vasospasm or other issues

Intraventricular hemorrhage

Traumatic brain and spinal cord injury

Central Nervous System Infections and Inflammations: Bacterial, tuberculous, fungal, parasitic meningitis, encephalitides, demyelinating syndromes, myelitis, motor neuron diseases

Peripheral Nervous Disorders, neuromuscular transmission disorders and myopathies: Acute polyneuropathies, chronic inflammatory neuropathies with relapses, boltulism, tick-bite paralysis, toxic neuropathies, diphtheria, porphyic neuropathy, myasthenia gravis, myasthenic syndrome, acute myopathies

Neurosurgical post-operative brain and spinal cases

Secondary Diseases of the Nervous System

Central Nervous System Disorders

Anoxic-Ischemic encephalopathy and cardioembolic strokes

Drug intoxications and poisonings

Endocrine disorders

Fat embolism

Fluid and electrolyte disturbances

Hyper and hypoglycemia

Hypertensive encephalopathies

Hyperthermia and hypothermia

Metabolic encephalopathies secondary of organ failure

Nutritional deficiencies

Pregnancy complications

Sepsis and SIRS-associated encephalopathy

Surgical complications

Vascular complications of hematological and autoimmune disorders

Withdrawal states

Peripheral Nervous System Disorders

Critical illness neuropathy, myopathy and neuromyopathy

Toxic and nutritional neuropathies and myopathies

Neuromuscular transmission disorders: drug-induced

brain injury (TBI) had a lower mortality rate when cared for in specialist neurocritical care centre compared with outcomes in non-neurocritical care hospitals. ${ }^{8}$ Several studies ${ }^{9-14}$ demonstrated that the regular use of guidelines wasassociated with improved outcomes: better Glasgow Outcome Scores (GOS), lower mortality and reduced ICU length of stay (LOS). Vespa and colleagues ${ }^{15}$ proved that continuous electroencephalogram (CEEG) monitoring of head injury patients shortened ICU LOS. Foley et $\mathrm{al}^{16}$ confirmed that specialized stroke units (intermediate care of similar discipline) reduced mortality, and were associated with ultimate greater independence and shorter hospital LOS for their patients.

2. Collaboration of disciplines is optimal. While specialized units staffed with individuals trained in both neurology/ neurosurgery and critical care seem ideal, they may be realistic only for a few Canadian centres at this time. Canadian institutions are best served by varied models rather than a single one, with designs determined by the complement of various specialists, their experience and collegial spirit. A collaborative model may be the best one for now and possibly the future, as no one type of specialist can study and manage all the varied problems alone.

Specialized neurological/neurosurgical involvement includes neuro-monitoring (with continuous EEG, evoked response testing, intracranial pressure monitoring, monitoring for vasospasm with transcranial Doppler, brain tissue oxygenation, cerebral microdialysis and other techniques), decompressive procedures, e.g., for stroke and trauma cases and innovative treatments for ischemic stroke, intraventricular hemorrhage and possibly intraparenchymal hemorrhage. While it can be acknowledged that expertise and understanding of neurological problems offers an advantage in caring for such patients, most of the complications of patients with head injury and subarachnoid hemorrhage (and probably other neurological and neurosurgical conditions that require intensive care) lie outside the nervous system, e.g., cardiovascular and respiratory failure. ${ }^{10}$ Treating such life-threatening systemic complications requires training and experience in critical care medicine.

Overlap of neurological/neurosurgical and ICU expertise include hypothermic treatment for comatose cardiac arrest patients, administration of anesthetic agents for refractory status epilepticus, managing "triple $\mathrm{H}$ " (hypertension, hypervolemia and hemodilution) therapy for treatment of vasospasm after subarachnoid hemorrhage and management of systemic infections, respiratory and other respiratory complications and co-morbidities in patients with conditions listed in the Table. Thus, many/most patients will benefit from care that includes expertise in both neurology/neurosurgery AND critical care medicine.

\section{Models of Neurocritical Care}

There are several possible models:

1. An NICU headed by a neurologist/neurosurgeon with fellowships/certification in both general and/or neurocritical care working with three to five similarly trained individuals. 2. An NICU with several general intensivists with formal neurointensive care training and experience.

3. Similarly trained single individuals as in 1 . and 2 . in a general medical-surgical-trauma ICU, rotating with general intensivists.

4. A neurosurgical ICU run by a neurosurgeon, but with close collegial support by intensivists/anesthetists and others, e.g., neurologists, infectious disease specialists, respiratory physicians, etc.

5. Neurologist-consultant in a general ICU: a neurologist who has a special interest in neurocritical care and who regularly consults on patients in general ICUs or special care units. 
The first two models or a combination of the two provide what should be the best models of specialized care for neurological and neurosurgical patients. Their infrastructure includes the physicians/surgeons, but also neurologically trained nurses and interconnections with other services as needed. However, the important aspects of specialized care can still be met with other models, provided planning, integration and dedication of individuals are in place.

It should be realized that some of these aspects may change with better manpower and resources. In some United States, German and Austrian centres a "critical mass" of neurointensivists, either from a neurological or critical care background, with an adequate complement of fellows and residents, seems to provide a self-sustaining model with adequate time for research, personal development, family time, etc. Most work in units devoted to acute disorders of the nervous system, and some do consultations in general ICUs. This model seems ideal; however, adequate staffing and cross-links to supportive specialists are essential. It hardly seems possible that neuro-intensivist truly be the master of diseases of the nervous system and the rest of the body, keeping up to date with the latest developments in acute lung injury, infection, changes in ventilation, sepsis, shock, multi-organ failure and other general ICU problems.

The model of the dedicated neurologist-consultant allows the individual to focus on disorders of the nervous system and leave the systemic management to dedicated intensivists. This requires co-operation, mutual respect and close collaboration. The advantage is that there is a more active neurologic presence in the ICU than previously existed in most centres. The neurologistconsultant provides prompt consultative service, daily follow-up, direction of neuro-monitoring tests and investigation, assistance in management, communication with families, and education of trainees and nurses in the unit. There is a learning curve for such neurologists to become proficient and to be accommodated within the ICU. The neurologist-consultant has limitations and needs to become familiar with systemic physiologic manipulation, advanced neuromonitoring specific to the ICU, various systemic diseases, drug interactions and altered metabolism. Thus, "information transfer" works in both directions, from the neurologist-consultant to the staff of the unit and from medical and paramedical ICU staff to the neurologist.

The intensivist with special training in neurocritical care has the advantage of working in an established system, with a "critical mass" in place. Intensivists are well-organized regionally and nationally and many are leaders in clinical research and epidemiology. Trials are vetted through a Clinical Trials Group of the Canadian Critical Care Society and, in the future, through the societies of the Canadian Neurosciences Foundation. This has successfully launched and completed many highly acclaimed studies. There is a network for collaboration across the country. The enhanced care for neurological and neurosurgical disorders would be a definite advance. Although this model has been in existence for some time in Europe, it is new to North America. Graduates of such one-two year programs do not have training and experience that is equivalent to a neurology or neurosurgical fellowship and post-fellowship training in subspecialty areas such as EEG, evoked potentials, EMG and neuroradiology.
A number of challenges, opportunities and threats face this young, evolving discipline. Which model is best? It seems reasonable that NICUs are appropriate for neurological hospitals, but in large, general ICUs the most appropriate model depends on the complement of intensivists, neuro-intensivists and specialized neurologists. The model will have to fit the complement of skill sets and manpower that is available.

\section{Remaining IsSUES}

The next decade will determine the way neurocritical care evolves in Canada. We should develop made-in-Canada solutions, but being aware of the issues and revisiting them will help this discipline to develop optimally. For now, it seems wise to work on an integrated model that makes use of the talents and training of the specialists in each centre. Some facilities may require special solutions, but on a national scale, integration and societal meetings will lead to advances in clinical research and practice guidelines as well as more rounded educational experiences for the clinician, the researcher and the trainee.

The formation of the Canadian Neurocritical Care Society will foster collaboration of neurologists, neurosurgeons and intensivists for research studies and training programs and will use operational models that seem to be working. Greater involvement by interested neurologists, pediatric neurologists and neurosurgeons is needed.

As can be seen in this review, no one can be the completely self-sufficient neuro-intensivist, fully equipped and up-to-date in all disciplines. A collaborative approach is needed for patient care and research; only this can lead to quality improvement with optimal patient outcomes and cost-savings, the principal objectives of this new field. Comprehensive patient-centred care, with appropriate guidelines and protocols, should form the foundation on which academic programs and further innovations can be based.

\section{REFERENCES}

1. Rincon F, Mayer SA. Neurocritical care: a distinct discipline? Curr Opin Crit Care. 2007;13:115-21.

2. The Canadian Neurocritical Care Group. Guidelines for the diagnosis of brain death. Can J Neurol Sci. 1999;26:64-6.

3. Jacka MJ, Zygun D (on behalf of the Canadian Neurocritical Care Society). Survey of management of severe head injury in Canada. Can J Neurol Sci. 2007;34:307-12.

4. Diringer MN, Edwards DF. Admission to a neurologic/neurosurgical intensive care unit is associated with reduced mortality rate after intracerebral hemorrhage. Crit Care Med. 2001;29: $635-40$.

5. Valeras PN, Eastwood D, Yun HJ, Spanaki MV, Hacein BL, Karsaris C, et al. Impact of a neurointensivist on outcomes in patients with head trauma treated in a neurosciences intensive care unit. J Neurosurg. 2006;104:713-19.

6. Elf K, Nilsson, Enblad P. Outcome after traumatic brain injury improved by an organized secondary insult program and standardized neurointensive care. Crit Care Med. 2002;30: 2129-34.

7. Patel HC, Menon DK, Tebbs S, Hawker R, Hutchinson PJ, Kirkpatrick P. Specialist neurocritical care and outcome from head injury. Intensive Care Med. 2002;28:547-53.

8. Patel HC, Bouamra O, Woodford M, King AT, Yates DW, Lecky FE, et al. Trends in head injury outcome from 1989 to 2003 and the effect of neurosurgical care: an observational study. Lancet. 2005;366:1538-44. 
9. Zygun DA, Doig CJ, Gupta AK, Whiting G, Nicholas C, Shepherd $\mathrm{E}$, et al. Non-neurological organ dysfunction in neurocritical care. J Crit Care. 2003;18:238-44.

10. Fakhry SM, Trask AL, Waller MA, Watts DD; IRTC Neurotrauma Task Force. Management of brain-injured patients by an evidence-based medicine protocol improves outcomes and decreases hospital charges. J Trauma. 2004:56:492-9.

11. Palmer S, Bader MK, Qureshi A, Palmer J, Shaver T, Borzatta M, et al. The impact on outcomes in a community hospital setting of using the AANS traumatic brain injury guidelines. Americans Associations for Neurologic Surgeons. J Trauma. 2001;50: 657-64.

12. Naredi S, Koskinen LO, Grande PO, Nordstrom CH, Nellgard B, Rydenhag B, et al. Treatment of traumatic head injury U.S./European guidelines or the Lund concept. Crit Care Med. 2003 Nov;31(11):2713-4.
13. Citerio G, Stocchetti N, Cormio M, Beretta L, Galli D, Pesenti A. Application of guidelines for severe head trauma: data from an Italian database. Eur J Emerg Med. 2003;10:68-72.

14. Bulger EM, Nathens AB, Rivara FP, Moore M, MacKenzie EJ, Jurkovich GJ; Brain Trauma Foundation. Management of severe head injury: institutional variation in care and effect on outcome. Crit Care Med. 2002;30:1870-6.

15. Vespa PM, Nenov V, Nuwer MR. Continuous EEG monitoring in the intensive care unit: early findings and clinical efficacy. J Clin Neurophysiol. 1999 Jan;16(1):1-13.

16. Foley N, Salter K, Teasell R. Specialized stroke services: a metaanalysis comparing three models of care. Cerebrovasc Dis. 2006; Dec 1;23(2-3): 194-202. 